\title{
Quantum Indeterminacy, Gauge Symmetries and Symplectic Reduction
}

\author{
Gabriel Catren ${ }^{* \dagger}$ \\ 1- Laboratoire SPHERE (UMR 7219), Université Paris Diderot-CNRS, Paris, France. \\ 2-Instituto de Filosofía “Dr. Alejandro Korn”, Facultad de Filosofía y Letras, Universidad de \\ Buenos Aires - CONICET, Buenos Aires, Argentina. \\ E-mail: gabrielcatren@gmail.com
}

As a consequence of Heisenberg indeterminacy principle, quantum states are defined by half the number of variables required in classical mechanics. The main claim of this paper is that this "reduction" in the number of variables required to completely describe a physical system can be understood as a consequence of the same formalism underlying the reduction procedure used in gauge theories, namely the Mardsen-Weinstein symplectic reduction. This fact points towards a gauge-theoretical interpretation of the indeterminacy principle in quantum mechanics.

Frontiers of Fundamental Physics 14

15-18 July 2014

Aix Marseille University (AMU) Saint-Charles Campus, Marseille, France

\footnotetext{
* Speaker.

${ }^{\dagger}$ The research leading to these results has received funding from the European Research Council under the European Community's Seventh Framework Programme (FP7/2007-2013 Grant Agreement n²63523, Project Philosophy of Canonical Quantum Gravity).
} 


\section{Introduction}

In general, the presence of symmetries entails a reduction in the amount of (invariant) information required to completely describe a physical system. One of the main examples of this relationship between symmetries and reduction is provided by the theory of constrained Hamiltonian systems (or gauge theories) [7]. In these theories, a classical system described by $2 n$ degrees of freedom and endowed with $k$ first-class constraints generating gauge symmetries can be reduced to a physical system described by $2(n-k)$ physical degrees of freedom. Now, the transition from classical to quantum mechanics also entails a reduction in the number of observables required to define a physical state, namely (in the simplest case) a reduction from $2 n$ classical observables $q$ and $p$ to the $n$ quantum observables $q$ or $p$. The fact that quantum states are defined modulo phase factors can be understood as a particular manifestation of such a reduction. In the simplest case, the phase invariance under translations in the position $q$ (i.e. the invariance modulo a phase factor) of an eigenstate $\left|p_{i}\right\rangle$ of the momentum operator

$$
q_{0} \cdot\left|p_{i}\right\rangle \mapsto e^{2 \pi i q_{0} p_{i}}\left|p_{i}\right\rangle \approx\left|p_{i}\right\rangle
$$

can be interpreted by saying that the position $q$ of the system described by the state $\left|p_{i}\right\rangle$ is completely "undetermined". In other terms, the possibility of changing the position without affecting the state can be rephrased by saying that the latter is not characterized by a well-defined value of the position. In this way, quantum phase symmetries encode the fact that quantum states are defined by half the number of variables required in classical mechanics. Heisenberg indeterminacy principle can be understood as a generalization of this reduction to more general states (such as for instance coherent states). The main stance of this paper is that, far from being a mere analogy, the quantum phase symmetries encoding the reduction from $2 n$ classical variables to $n$ quantum variables can be understood as a consequence of the same geometric formalism underlying the gauge symmetries, namely the symplectic reduction procedure (see also Refs.[2, 3, 4]). In what follows, we shall focus on the conceptual aspects of the arguments and we shall omit important mathematical details (a more detailed presentation can be found in Ref.[5]).

\section{The Moment Map}

Let $M$ be a connected manifold endowed with a symplectic structure $\omega$ [1]. The symplectic structure $\omega$ permits to define a map

$$
\begin{aligned}
\omega: \text { Observables } & \rightarrow \text { Classical operators } \\
f & \mapsto v_{f},
\end{aligned}
$$

between observables-i.e. functions $f \in \mathscr{C}^{\infty}(M)$ on $M$-and classical operators-i.e. the so-called Hamiltonian vector fields $v_{f}$ on $M$-. It is worth stressing that this correspondence between observables assigning numbers to states and operators acting on states, far from being a characteristic feature of quantum mechanics, is at the heart of classical mechanics (see Refs. [2, 3, 4] for a discussion of this point).

Let's suppose now that $M$ is endowed with an action of a Lie group $G$ preserving $\omega$. As it is the case with every group action on a manifold, such an action is implemented at the infinitesimal level 
by some vector fields on $M$ called fundamental vector fields. More precisely, there is a map that sends each element $X$ in the Lie algebra $\mathfrak{g}$ of $G$ to a fundamental vector field $v_{X}$ on $M$. The action is called Hamiltonian if these fundamental vector fields can be obtained via the correspondence (2.1), i.e. if each fundamental vector field can be derived from an observable (called generating function of the group action) by means of the map (2.1) defined by $\omega$. Briefly, the action is Hamiltonian if the vector fields on $M$ that generate the group action can be derived from a generating function. This means that given a Hamiltonian action we can define a map

$$
\tilde{\mu}: \mathfrak{g} \rightarrow \mathscr{C}^{\infty}(M)
$$

sending each Lie algebra element $X \in \mathfrak{g}$ to a generating function $f_{X}$ such that the classical operator defined by the latter via (2.1) is the fundamental vector field $v_{X}$ on $M$ associated to $X$. This map is called co-moment map and was introduced by the French mathematician Jean-Marie Souriau [11]. In this way, a Hamiltonian action on a symplectic manifold entails the existence of a privileged subalgebra of the algebra of observables on $M$, namely the observables given by the generating functions of the group action.

In what follows we shall also need a map dual to the co-moment map. Both $\mathfrak{g}$ and $\mathscr{C}^{\infty}(M)$ in (2.2) have dual structures, namely the linear dual $\mathfrak{g}^{*}$ and $M$ respectively. On the one hand, $\mathfrak{g}$ and $\mathfrak{g}^{*}$ are dual in the sense that there is a bilinear map $\langle\cdot, \cdot\rangle: \mathfrak{g} \times \mathfrak{g}^{*} \rightarrow \mathbb{R}$. On the other hand, the symplectic manifold $M$ and the algebra of observables $\mathscr{C}^{\infty}(M)$ are dual in the sense that there is an evaluation map $\mathscr{C}^{\infty}(M) \times M \rightarrow \mathbb{R}$ given by $(f, m) \mapsto f(m)$. By means of these dualities we can introduce a map (called moment map)

$$
\mu: M \rightarrow \mathfrak{g}^{*}
$$

which is dual to the co-moment map (2.2). Given the moment map, the co-moment map can be recovered by means of the following expression

$$
\tilde{\mu}(X)(m)=f_{X}(m)=\langle X, \mu(m)\rangle,
$$

for $X \in \mathfrak{g}$ and $m \in M$.

In what follows, a symplectic manifold endowed with a Hamiltonian $G$-action will be called Hamiltonian G-manifold and denoted $(M, \omega, G)$. The simplest example of this formalism is given by the action of the abelian group $G=\mathbb{R}$ on $M=\{(q, p)\}$ by translations in the position $q$. In this case, the moment map is simply given by $\mu:(q, p) \mapsto p$. In turn, the co-moment map is given by $\tilde{\mu}: q_{0} \mapsto f_{q_{0}}$ (where $q_{0}$ is the coordinate of the 1-dimensional vector space $\mathfrak{g}$ ) such that $f_{q_{0}}(q, p)=\left\langle q_{0}, \mu(q, p)\right\rangle=q_{0} p$. The classical operator associated to this generating function via (2.1) is $v_{f_{q_{0}}}=q_{0} \frac{\partial}{\partial q}$.

\section{Kirillov's Orbit Method and Symplectic Reduction}

When a group action on a symplectic manifold $M$ is Hamiltonian there is a relationshipestablished by the moment map $\mu$-between classical states in $M$ and elements in the dual $\mathfrak{g}^{*}$ of the Lie algebra $\mathfrak{g}$. Now, how can we understand this relationship in conceptual terms? One possible answer to this question is provided by Kirillov's orbit method [8]. According to this method (which only works for certain Lie groups $G$ ), $\mathfrak{g}^{*}$ encodes (what we could call) the unitary representation 
theory of $G$. Let's unpack this statement. Firstly, $\mathfrak{g}^{*}$ has the structure of a Poisson manifold endowed with an action of $G$ (the so-called coadjoint action). Secondly, it is a well-known fact in the theory of Poisson manifolds that a Poisson manifold can be foliated in symplectic manifolds. In particular, $\mathfrak{g}^{*}$ can be understood as a family of classical systems (that we shall denote $\mathscr{O}$ ) described by the symplectic leaves of $\mathfrak{g}^{*}$. Now, the remarkable fact regarding the Poisson manifold $\mathfrak{g}^{*}$ is that the orbits of the $G$-action are exactly the symplectic leaves of $\mathfrak{g}^{*}$. In other terms, each classical system $\mathscr{O}$ in $\mathfrak{g}^{*}$, being an orbit of the $G$-action on $\mathfrak{g}^{*}$, is endowed with a transitive action of $G .{ }^{1}$ The transitivity of the $G$-action means that the classical systems $\mathscr{O}$ in $\mathfrak{g}^{*}$ are irreducible with respect to the $G$-action. In other terms, there are no $G$-invariant subspaces within a classical system $\mathscr{O}$ in $\mathfrak{g}^{*}$. Now, the main idea of Kirillov's orbit method is that the quantization of the G-irreducible classical systems $\mathscr{O}$ yields quantum systems that are unitary irreducible representations (unirreps in short) of $G$. In other terms, the process of quantization defines a map

$$
\mathscr{O} \rightsquigarrow \mathscr{H}_{\mathscr{O}},
$$

between the $G$-irreducible classical systems $\mathscr{O}$ in $\mathfrak{g}^{*}$ and Hilbert spaces $\mathscr{H}_{\mathscr{O}}$ carrying unirreps of $G$. In the cases for which the orbit method works, any unirrep of $G$ can be obtained by quantizing an irreducible classical system $\mathscr{O}$ in $\mathfrak{g}^{*}$. Since any unitary representation of $G$ can be decomposed as a direct sum of unirreps, the Poisson manifold $\mathfrak{g}^{*}$ encodes the unitary representation theory of $G$.

In what follows, we shall only consider the case of an abelian Lie group $G$. In this case, the irreducible classical systems in $\mathfrak{g}^{*}$ are just points. In other terms, the $G$-action on $\mathfrak{g}^{*}$ leaves its points invariant, i.e. each point is an orbit. A point $\xi \in \mathfrak{g}^{*}$ defines a 1-dimensional unirrep $\rho_{\xi}$ of $G$ given by

$$
\begin{aligned}
\rho_{\xi}: G & \rightarrow U(1) \\
e^{X} & \mapsto e^{2 \pi i\langle\xi, X\rangle}, \quad X \in \mathfrak{g} .
\end{aligned}
$$

The quantization of the one-point classical system $\xi \in \mathfrak{g}^{*}$ yields a 1-dimensional Hilbert space $\mathscr{H}_{\xi}$ containing a unique $U(1)$-class of normalized vectors. The unitary $G$-action on these vectors is implemented by means of the phase factors in $U(1)$ defined by the unirrep (3.1). If we assume that quantum states are define modulo overall phase factors, the resulting quantum theory is composed of a unique $(G, \xi)$-phase invariant quantum state that we shall denote $|\xi\rangle$.

We have thus far argued that $\mathfrak{g}^{*}$ can be understood as a family of $G$-irreducible classical systems encoding the unitary representation theory of $G$. Let's consider now the quantization of the Hamiltonian $G$-manifold $M$. Analogously to the case of the classical systems in $\mathfrak{g}^{*}$, we expect the quantization of $M$ to yield a Hilbert space $\mathscr{H}_{M}$ carrying a unitary representation of $G$. However, $M$ is not necessarily $G$-irreducible, i.e. the action of $G$ on $M$ is not necessarily transitive. Therefore, there is no reason to expect the unitary representation of $G$ carried by $\mathscr{H}_{M}$ to be irreducible. Now, the representation space $\mathscr{H}_{M}$ can be decomposed (as every unitary representation) as a direct sum of the unirreps of $G$, i.e. as a direct sum of the Hilbert spaces $\mathscr{H}_{\mathscr{O}}$ obtained by quantizing the $G$-irreducible classical systems in $\mathfrak{g}^{*}$. In other terms, we have a decomposition of the form

$$
\mathscr{H}_{M}=\bigoplus_{\mathscr{O} \subset \mathfrak{g}^{*}} m(\mathscr{O}, M) \mathscr{H}_{\mathscr{O}}
$$

\footnotetext{
${ }^{1}$ The transitivity of the action means that any two points in $\mathscr{O}$ can always be connected by means of a group element in $G$.
} 
where $m(\mathscr{O}, M)$ is the number of times (i.e. the multiplicity) that the unirrep $\mathscr{H}_{\mathscr{O}}$ occurs in $\mathscr{H}_{M}$.

If we assume that we know the unirreps $\mathscr{H}_{\mathscr{O}}$, expression (3.2) means that the multiplicities $m(\mathscr{O}, M)$ completely define the quantization of the Hamiltonian $G$-manifold $M$. Now, how can we calculate these multiplicities? In Ref.[6], Guillemin and Sternberg conjectured that the moment map $\mu: M \rightarrow \mathfrak{g}^{*}$ encodes the information concerning the unirreps of $G$ that occur in $\mathscr{H}_{M}$ (and the corresponding multiplicities). Roughly speaking, the idea is that we can construct the decomposition (3.2) by "pulling-back" the unirreps $\mathscr{H}_{\mathscr{O}}$ to $M$ by means of $\mu$. Let's describe how this works in the abelian case by calculating the multiplicity in $\mathscr{H}_{M}$-where $(M, \omega, G)$ is a Hamiltonian $G$ manifold-of the 1-dimensional unirrep $\mathscr{H}_{\xi}$ defined by the (one-point) irreducible classical system $\xi \in \mathfrak{g}^{*}$. To do so, we have to consider the subset of classical states in $M$ that the moment map $\mu$ puts in correspondence to the irreducible classical system $\xi$, i.e. the subset

$$
\mu^{-1}(\xi) \subset M
$$

In a first rough approach, we could guess that the quantization of $\mu^{-1}(\xi)$ would yield the term $m(\xi, M) \mathscr{H}_{\xi}$ in the decomposition (3.2). Since the moment map is assumed to be $G$-equivariantin the sense that $\mu(g \cdot m)=g^{-1} \cdot \mu(m)$-, the preimage $\mu^{-1}(\xi)$ is a $G$-space, i.e. a collection of $G$-orbits. We could then guess that the quantization of these collection of $G$-orbits would give one copy of $\mathscr{H}_{\xi}$ for each $G$-orbit, thereby yielding the term $m(\xi, M) \mathscr{H}_{\xi}$ in (3.2). However, this idea does not work for a fundamental reason, namely that the preimage $\mu^{-1}(\xi)$ is not in general a symplectic manifold, which means that it cannot be quantized. Now, a fundamental result in symplectic geometry is that, under nice conditions, the quotient $M_{\xi} \doteq \mu^{-1}(\xi) / G$ is a symplectic manifold. This is the content of the so-called Mardsen-Weinstein symplectic reduction procedure [10]. In what follows, the procedure of passing from $M$ to $M_{\xi}$ will be called $\xi_{\text {-symplectic reduction }}$ and the symplectic manifold $M_{\xi}$ will be called $\xi$-symplectic quotient.

Guillemin and Sternberg conjectured in Ref.[6] (and demonstrate it for a particular case) that the quantization of the $\xi$-symplectic quotient $M_{\xi}$ yields a Hilbert space $\mathscr{H}_{M_{\xi}}$ satisfying the following property: each state in $\mathscr{H}_{M_{\xi}}$ defines a map of Hilbert spaces $\mathscr{H}_{\xi} \rightarrow \mathscr{H}_{M}$ intertwining the unitary $G$-action on both spaces (see Ref.[5] for a conceptual discussion of this result). This implies that the number of independent states in $\mathscr{H}_{M_{\xi}}$ (i.e. its dimension) gives the number of independent copies of $|\xi\rangle$ in $\mathscr{H}_{M}$, i.e. the multiplicity $m(\xi, M)$. In this way, whereas the quantization of the (trivial) classical system $\xi$ belonging to the image of $\mu_{M}$ yields the unirreps $\mathscr{H}_{\xi}$ that occur in the decomposition (3.2) of $\mathscr{H}_{M}$, the quantization of the $\xi$-symplectic quotients $M_{\xi}$ yields the multiplicities $m(\xi, M)$. Therefore, the 1-dimensional unirrep $\mathscr{H}_{\xi}$ occurs in $\mathscr{H}_{M}$ if $\mathscr{H}_{M_{\xi}} \neq \emptyset$. This is the case if $M_{\xi} \neq \emptyset$, that is if $\xi$ is in the image of $\mu$. In particular, if $M_{\xi}$ is a one-point space, the Hilbert space $\mathscr{H}_{M_{\xi}}$ is composed of a unique quantum state. Then, $m(\xi, M)=1$, i.e. the unirrep $\mathscr{H}_{\xi}$ occurs only once in $\mathscr{H}_{M}$.

\section{Quantization Commutes with Reduction}

We shall now consider a gauge theory associated to a Hamiltonian $G$-manifold $(M, \omega, G)$, where $G$ is not necessarily abelian. A theory on $(M, \omega, G)$ is called a gauge theory if the Hamilton equations "constraint" the solutions to be in the constraint surface $\mu^{-1}(0) \subset M$ defined by the value $0 \in \mathfrak{g}^{*}$ of the moment map [9]. In this case, the group $G$ is called the gauge group of the theory 
(and denoted $G_{\text {gau }}$ ) and the generating functions $f_{i}(m)=\left\langle\mu(m), X_{i}\right\rangle$ (for $X_{i} \in \mathfrak{g}$ ) defined by the comoment map are called constraints. The reason is that the constraint surface can be equivalently defined by means of the constraint equations $f_{i}(m)=0$. The Mardsen-Weinstein reduction theorem states that the 0 -symplectic quotient

$$
M_{0} \simeq \mu^{-1}(0) / G_{\text {gau }}
$$

(also called reduced phase space in the physics literature) is a symplectic manifold.

Now, the quantization of the irreducible classical system $0 \in \mathfrak{g}^{*}$ yields a 1-dimensional Hilbert space (whose unique quantum state is denoted $|0\rangle$ ) carrying the trivial unirrep of $G$. In this case, the Guillemin-Sternberg conjecture states that each state $\psi_{a}$ in a basis of $\mathscr{H}_{M_{0}}$ defines a map $\varphi_{a}$ : $|0\rangle \rightarrow \mathscr{H}_{M}$. Since this map intertwines the $G$-action, the image of $\varphi_{a}$ in $\mathscr{H}_{M}$ must be a $G$-invariant state. All in all, a basis of $\mathscr{H}_{M_{0}}$ defines a set of independent $G$-invariant states in $\mathscr{H}_{M}$. We can rephrase this result by means of the following isomorphism

$$
\mathscr{H}_{M_{0}} \simeq \mathscr{H}_{M}^{G_{\text {gau }}}
$$

where $\mathscr{H}_{M}^{G_{\text {gau }}}$ denotes the Hilbert space generated by the $G_{\text {gau }}$-invariant quantum states on $M$. This means that the Hilbert space $\mathscr{H}_{M_{0}}$ obtained by quantizing the 0 -symplectic quotient $M_{0}$ defined by the irreducible classical system $0 \in \mathfrak{g}^{*}$ is in bijective correspondence with the Hilbert space $\mathscr{H}_{M}^{G_{\text {gau }}}$ obtained by selecting the $G_{g a u}$-invariant quantum states in $\mathscr{H}_{M}$. Diagramatically,

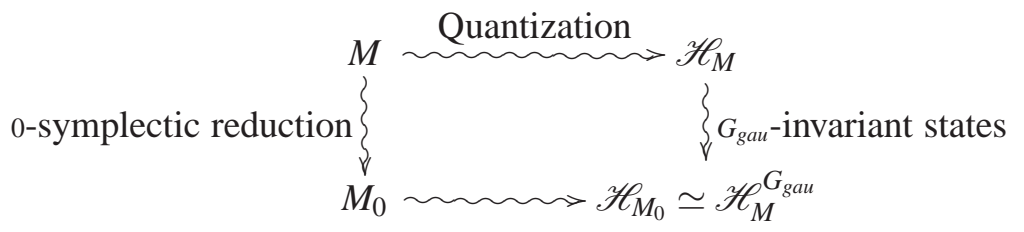

The commutativity of this diagram guarantees the validity of the so-called Dirac's method for quantizing gauge theories [7]. Indeed, the commutativity of (4.3) guarantees that one can either reduce the classical theory defined by the original phase space $M$ (which is in general problematic) and quantize the reduced phase space $M_{0}$ afterwards or directly quantize $M$ and then select the $G_{\text {gau }}{ }^{-}$ invariants quantum states in $\mathscr{H}_{M}$ (Dirac's method). The $G_{\text {gau }}$-invariant quantum states in $\mathscr{H}_{M}$ are the states that satisfy the quantum constraint equations $\hat{f}_{i} \psi=0$, where $\hat{f}_{i}$ is the quantum operator associated to the constraint $f_{i}$ [7]. The point that we want to stress here is that the $G_{\text {gau }}$-invariance of the quantum states of a gauge theory is the quantum counterpart of the classical symplectic reduction with respect to the irreducible classical system 0 defining the trivial unirrep of $G$. We could say that the selection of the $G$-invariant quantum states in $\mathscr{H}_{M}$ implements the notion of 0 -reduction at the quantum level. Therefore, we can rephrase (4.2) by saying that "quantization commutes with 0-reduction".

\section{On the Quantum Indeterminacy as a Form of Symplectic Reduction}

Now, we are here interested in ordinary theories (i.e. theories that are not gauge theories) defined by Hamiltonian $G$-manifolds $(M, \omega, G)$. In other terms, we want to consider symplectic manifolds endowed with a Hamiltonian $G$-action such that the corresponding moment map $\mu$ is 
not constrained to a unique value in $\mathfrak{g}^{*}$. In these cases, the group $G$ will be called phase group and the (non-constrained) generating functions $f_{i}$ phase observables. Differently from the constraints of a gauge theory (which restrict the theory to the trivial unirrep of the gauge group $G_{\text {gau }}$ ), the phase observables $f_{i}$ do not select a single unirrep of the phase group $G$. Therefore, while the action of a gauge group defines a unique 0 -symplectic quotient (4.1) associated to the trivial unirrep of $G_{\text {gau }}$, the action of a phase group defines a different $\xi$-symplectic quotient

$$
M_{\xi} \doteq \mu^{-1}(\xi) / G
$$

for each unirrep $\xi \in \mathfrak{g}$ of the phase group $G$ (where $G$ is supposed to be abelian). In this way, the phase $G$-action on $M$ entails the existence of a whole set of $\xi$-symplectic quotients $M_{\xi}$. In the case given by the action of $\mathbb{R}$ on $M=\{(q, p)\}$ by translations in $q$, each value $p_{i}$ of the moment map $\mu:(q, p) \mapsto p$ defines a $p_{i}$-symplectic quotient $M_{p_{i}} \doteq \mu^{-1}\left(p_{i}\right) / G$ containing a unique point.

In gauge theories, the bijection (4.2) implies that the quantization of the unique symplectic quotient $M_{0}$ yields the $G$-invariant quantum states in $\mathscr{H}_{M}$. Now, what is the analogue of this bijection in the case of the $\xi$-symplectic quotients $M_{\xi}$ ? In other terms, what kind of states in $\mathscr{H}_{M}$ do we obtain if we quantize the $\xi$-symplectic quotients $M_{\xi}$ ? It can be shown that in the abelian case the generalization of the bijection (4.2) is given by the following isomorphism (see Ref.[5] for details)

$$
\mathscr{H}_{M_{\xi}} \simeq \mathscr{H}_{M}^{(G, \xi)} .
$$

Here, $\mathscr{H}_{M}^{(G, \xi)}$ denotes the Hilbert space containing the $(G, \xi)$-phase invariant states in $\mathscr{H}_{M}$, i.e. the states $|\xi, \ldots\rangle$ that are invariant modulo overall phase factors in $U(1)$ defined by the 1-dim. unirrep $\rho_{\xi}$ of $G$ defined by $\xi$ :

$$
\begin{aligned}
\rho_{\xi}: G & \rightarrow U(1) \\
e^{X} & \mapsto e^{2 \pi i\langle\xi, X\rangle}, \quad X \in \mathfrak{g} .
\end{aligned}
$$

In this way, the quantization of the $\xi$-symplectic quotient $M_{\xi}$ does not yield the $G$-invariant states in $\mathscr{H}_{M}$, but rather the $(G, \xi)$-phase invariant states. The corresponding commuting "diagram" is now:

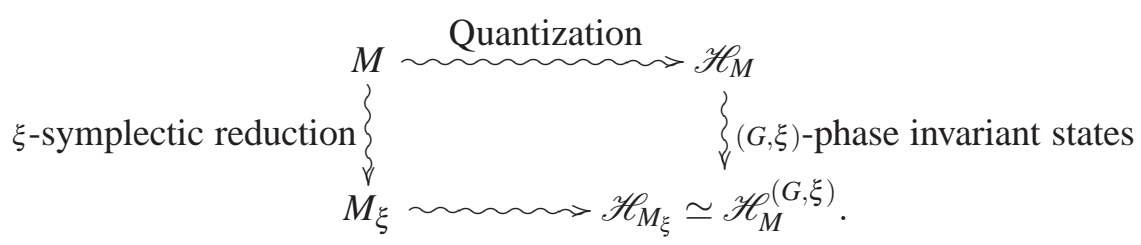

We can now claim that "quantization commutes with $\xi$-reduction" provided that the notion of $\xi$-reduction at the quantum level is given by the selection of the $(G, \xi)$-phase invariant quantum states in $\mathscr{H}_{M}$ (instead of the $G_{\text {gau }}$-invariant states as it is the case in gauge theories). The important result is that the $(G, \xi)$-phase invariance of quantum states is the quantum counterpart of the symplectic reduction with respect to the non-zero value $\xi$ of the moment map. This means that quantum phase invariance is the generalization of the strict $G$-invariance appearing in gauge theories to the cases of $\xi$-symplectic reductions with $\xi \neq 0$. 
Now, the $(G, \xi)$-phase invariance of the states $|\xi, \ldots\rangle$ means that the variable acted upon by $G$ plays no role in the definition of $|\xi, \ldots\rangle$. In other terms, we can change the value of this variable without changing the state $|\xi, \ldots\rangle$ as such (i.e. modulo an overall phase factor). We can then say that the states $|\xi, \ldots\rangle$ are such that the variable acted upon by $G$ is completely "undetermined". In the case of the group of translations in $q$ acting on $M=\{(q, p)\}$, the quantization of the one-point $p_{i^{-}}$ symplectic quotient $M_{p_{i}}$ defined by the value $p_{i}$ of the momentum yields a 1-dimensional Hilbert space $\mathscr{H}_{M_{p_{i}}}$ containing a unique quantum state. By using the bijection (5.1), this state defines a unique $\left(G, p_{i}\right)$-phase invariant quantum state $\left|p_{i}\right\rangle$ in $\mathscr{H}_{M}$. According to (5.2), a translation in $q_{0}$ of the state $\left|p_{i}\right\rangle$ just multiplies the state by an overall phase factor of the form $e^{2 \pi i q_{0} p_{i}}$. This means that the position $q$ of the state $\left|p_{i}\right\rangle$ can be modified without changing the state as such. In more usual terms, the position $q$ of the state $\left|p_{i}\right\rangle$ is completely "undetermined". In this way, the phase invariance of quantum states encodes the extreme cases of Heisenberg indeterminacy principle, namely the cases given by a well-determined variable and a completely undetermined conjugate variable. The important point that we want to stress here is that this phase invariance is the generalization of the strict invariance relevant in gauge theories to the cases in which the corresponding classical symplectic reduction takes place with respect to non-zero values of the moment map.

According to what we have just said, the essential difference between a gauge theory and an ordinary theory endowed with a Hamiltonian group action is the following: while the quantum states of a gauge theory are necessarily $G_{g a u}$-invariant (i.e. states transforming in the trivial unirrep of the gauge group), a non-constrained Hamiltonian theory contains states that are $G$-invariant modulo phase factors (i.e. states transforming in non-trivial unirreps of $G$ ). This difference has an important consequence regarding the possibility of breaking the corresponding symmetries. In the quantum theory obtained by quantizing a non-constrained Hamiltonian $G$-manifold $(M, \omega, G)$, one can superpose different $(G, \xi)$-phase invariant states carrying different unirreps (5.2). In other terms, $\mathscr{H}_{M}$ contains states of the form $|\psi\rangle=\sum_{\xi} \psi(\xi)|\xi, \ldots\rangle$. Now, a state $|\psi\rangle$ obtained by superposing $(G, \xi)$-phase invariant states is no longer $G$-phase invariant. Indeed, the action of $G$ changes the relative phases between the terms $|\xi, \ldots\rangle$ of the superposition. This means that the superposition of states transforming under different unirreps of $G$ breaks the G-phase symmetry, i.e. the indeterminacy in the variable acted upon by $G$. In other terms, the introduction of an indeterminacy in the variable $\xi$ that labels the unirreps of $G$ breaks the complete indeterminacy in the (conjugate) variable acted upon by $G$. On the contrary, the quantization of a gauge theory only contains $G_{g a u}$-invariant states, i.e. states transforming in the trivial unirrep of $G_{g a u}$. Therefore, it is not possible to superpose states carrying different unirreps of the gauge group. Hence, no quantum state breaks the gauge symmetry. To sum up, we can say that while the quantum gauge symmetries are not broken by the physical states, the quantum phase symmetries can be broken by superposing states transforming in different unirreps of the phase group.

We can also understand the fact that the position $q$ does not define a well-determined property of an eigenstate $\left|p_{i}\right\rangle$ of the momentum operator in gauge-theoretical terms as follows. In gauge theories, only the observables $f \in \mathscr{C}^{\infty}(M)$ whose restriction to the constraint surface $\mu^{-1}(0)$ is $G_{\text {gau }}$-invariant (i.e. the so-called Dirac observables) define observables on $M_{0}$. In turn, only the observables on $M_{0}$ can be used to define properties of the quantum states obtained by quantizing $M_{0}$, which are in correspondence, via the bijection (4.2), with the $G$-invariant states in $\mathscr{H}_{M}$. In the 
case of the ordinary (i.e. non-gauge) theory given by the Hamiltonian action of the group $G$ of translations in $q$ acting on $M=\{(q, p)\}$, the observable $q$ is not $G$-invariant. Hence, the position $q$ is not a Dirac observable, i.e. it does not define an observable on the one-point $p_{i}$-symplectic quotients $M_{p_{i}}$ (for the different values of $p_{i}$ ). Consequently, the position $q$ cannot define a property of the quantum state $\left|p_{i}\right\rangle$ defined by the trivial quantization of $M_{p_{i}}$. According to the conceptual framework proposed in this article, this is just a gauge-theoretical restatement of the fact that the position $q$, far from defining a property of the state $\left|p_{i}\right\rangle$, is completely undetermined.

From a conceptual viewpoint, the fact that the Dirac observables of a gauge theory do not single out a particular representative in each gauge orbit means neither that the theory is "incomplete" (i.e. that it might be possible to find some hypothetical "hidden variables" capable of establishing a physical distinction between gauge-equivalent elements in a gauge orbit), nor that there is some form of epistemic restriction to the amount of information an observer can have about a gauge system. According to the so-called Dirac conjecture [7], Dirac observables cannot distinguish between elements belonging to the same gauge orbit just because these elements are different representations of the same physical state. Now, the fact that quantum phase symmetries can be understood as a generalization (to the non-zero values of the moment map) of gauge symmetries points towards what we could characterize as a gauge-theoretical interpretation of the indeterminacy relations in quantum mechanics. In other terms, the indeterminacy in the position $q$ of the state $\left|p_{i}\right\rangle$ should not be interpreted as a form of theoretical incompleteness or epistemic limitation, but rather as a consequence of the same group-theoretical reduction mechanism which is at work in gauge theories. In this way, the problem of providing a satisfactory conceptual interpretation of the quantum indeterminacy is reduced to the (open) problem of understanding the rationale behind gauge symmetries.

\section{References}

[1] R. Abraham and J.E. Marsden, Foundations of Mechanics, 2nd edn., Addison-Wesley Publishing Company, Reading (1978).

[2] G. Catren, On classical and quantum objectivity, Foundations of Physics 38, 470-487 (2008).

[3] G. Catren, Can classical description of physical reality be considered complete?, in M. Bitbol, P. Kerszberg, and J. Petitot (eds.) Constituting Objectivity: Transcendental Perspectives on Modern Physics, The Western Ontario Series in the Philosophy of Science, vol. 74, 375-386, Springer-Verlag, Berlin (2009).

[4] G. Catren, Quantum ontology in the light of gauge theories, in C. de Ronde, S. Aerts, and D. Aerts (eds.) Probing the Meaning of Quantum Mechanics: Physical, Philosophical, and Logical Perspectives, World Scientific Publishing (2014).

[5] G. Catren, On the Relation Between Gauge and Phase Symmetries, Foundations of Physics, vol. 44, Issue 12, 1317-1335 (2014).

[6] V. Guillemin and S. Sternberg, Geometric quantization and multiplicities of group representations, Invent. math. 67, 515-538.

[7] M. Henneaux and C. Teitelboim, Quantization of Gauge Systems, Princeton University Press, New Jersey (1994). 
[8] A.A. Kirillov, Lectures on the Orbit Method, Graduate Studies in Mathematics, vol. 64, AMS, Providence (2004).

[9] B. Kostant and S. Sternberg, Symplectic Reduction, BRS Cohomology, and Infinite Dimensional Clifford Algebras, Annals of Physics 176, 49-113 (1987).

[10] J.E. Marsden and A. Weinstein, Reduction of symplectic manifolds with symmetry, Rep. Math. Phys. 5, 121-130 (1974).

[11] J.-M. Souriau, Structure of Dynamical Systems. A Symplectic View of Physics, Birkhäuser, Boston (1997). 